\title{
Pilot Study, Does It Really Matter? Learning Lessons from Conducting a Pilot Study for a Qualitative PhD Thesis
}

\author{
Nashwa Ismail (Corresponding author) \\ University of Bath- Department of Education, UK \\ E-mail: n.ismail@bath.ac.uk \\ Gary Kinchin \\ University of Southampton, Southampton Education School, UK \\ E-mail: G.D.Kinchin@soton.ac.uk \\ Julie-Ann Edwards \\ University of Southampton, Southampton Education School, UK \\ E-mail: J.S.Edwards@soton.ac.uk
}

Received: August 17, 2017 Accepted: September 20, 2017 Published: November 6, 2017

doi:10.5296/ijssr.v6i1.11720 URL: http://dx.doi.org/10.5296/ijssr.v6i1.11720

\begin{abstract}
A Pilot Study (PS) is a small-scale research project conducted before the final full-scale study. A PS helps researchers to test in reality how likely the research process is to work, in order to help them decide how best to conduct the final research study. In piloting a study, a researcher can identify or refine a research question, discover what methods are best for pursuing it, and estimate how much time and what resources will be necessary to complete the larger final version of the study. There is, however, a paucity in literature that focuses on using, reading and representing PSs. This article discusses the importance of a PS to test and identify how methods and ideas would work in practice when undertaking a qualitative $\mathrm{PhD}$ thesis. The proposed PS in this paper addressed many challenges, and the researcher reflected on different perspectives of their work including ethical, cultural, social and professional issues. By the end
\end{abstract}


of the $\mathrm{PhD}$ thesis, undertaken with the guidance of the PS, it was found that a well-conducted PS, giving a clear list of aims and objectives within a formal framework, can encourage methodological rigour and ensure the validity of both the study itself and the methodology applied. The objectives of this paper are thus threefold: first, to identify the issues within a specific qualitative PS prior to conducting the final study; second, to address the researcher's reflections on these findings and finally, to share the experiences and knowledge that a PS can be expected to bring.

Keywords: pilot study, final study, qualitative, flexibility, validity

\section{Introduction}

PSs guide the development of the research plan (Prescott \& Soeken, 1989), as the smaller (pilot) study informs and gives feedback to the larger (final) study. Based on this feedback, the researcher can make adjustments to and refine the research methodology before attempting the final study. This article presents the author's reflection on the lessons learned from a PS that was recently used in completing a $\mathrm{PhD}$ qualitative thesis on investigating the perceptions of online tutors with regards to student-centred learning (SCL) in Egyptian higher education. The purpose of the $\mathrm{PhD}$ research study was to investigate Egyptian tutors' understanding and implementation of SCL as a concept in the online learning (OL) environment, and to examine the factors that influence these perceptions. The study is phenomenologically qualitative and the instruments used for collecting data are focus groups and semi-structured interviews. The PS conducted before the final study was intended to explore any methodological issues arising from putting the methodology into in practice. By showing how the relevant aspects of the main study changed as a result of conducting the PS, the comparison between the methodology before and after conducting the PS proves the value of conducting a PS in qualitative research. According to Teijlingen et al. (2002), researchers often claim that they learned from conducting a PS and made necessary changes, but do so without offering the reader details on what exactly was learnt. This is yet another essential reason for this paper, in which the lessons learned and the reflections of the researcher are addressed in detail.

The structure of this article is as follows: first, it reviews contributions to research which address the usefulness of PSs, and discusses the reasons that PSs are scarce in the research literature, or scarcely reported. This discussion places the focus on the value of PSs and their roles in qualitative rather than in quantitative research. It is followed by a brief description of the PS conducted for the $\mathrm{PhD}$ project on which the discussion in this article is based. It then presents the researcher's professional reflections on conducting this PS, summarising the lessons learned. The final section concludes with recommendations for conducting PSs and addresses some related issue for future research interests.

\subsection{Why Pilot Studies?}

PSs are likely to be 'under-discussed, underused and underreported' (Prescott \& Soeken, 1989, p. 60). According to Teijlingen and Hundley (2001), full reports on PSs are rare in research literature, and even when reported, they are often used to justify the research methods or use of a particular research tool, but mainly focusing on quantitative methods. This draws attention to 
the fact that there might be a tendency to assume that the role of pilot works consists of only the testing of research instruments (Sampson, 2004), which might lead us to think that PSs are of limited value. Examples of this understanding of the benefits of PSs as being limited include Baker (1994), who explains that piloting consists exclusively of pre-testing a particular research instrument, and to give the researcher advance warning on where the main research project might fail. Similarly, Crossman (2007) sees only limited benefits in a PS, which can help in estimating the required time and costs for the project. A wider range of benefits is acknowledged by Prescott and Soeken (1989) and Teijlingen and Hundley (2001), who extensively discuss the different possible benefits of PSs and provide ideas about potential obstacles which might occur in the main data collection phase, including in data collection method or sample recruitment strategy, Teijlingen et al. (2001) and Teijlingen and Hundley (2001) both provide a summary of the reasons for performing a PS for both quantitative and qualitative methods; these reasons range from process and management to resources and the scientific process. It is worth noting that benefits are only for scientific research and there are no benefits for the researchers themselves from a professional perspective including research informed practice, leadership and critical thinking.

According to Janghorban et al. (2014), Kim (2010), Padgett (2008), and Prescott and Soeken (1989), there is a measurable lack of research on PSs in general, and on PSs in qualitative research in particular. In order to widen the range of literature on PSs examined, particularly literature on qualitative studies, a search through various journals was conducted, but indeed the research literature found mostly focused on quantitative studies, for example literature from US National Institutes of Health's National Library of Medicine with its numerous articles focused on sharing research and researchers' experience in health and nursing related issues. In terms of qualitative research, there was limited focus in journals such as Qualitative Social Work (QSW), Qualitative Inquiry and Qualitative Health Research. Moreover, few academics addressed the usefulness of PS for the competence of qualitative researchers, and there also does not seem to be any textbook in existence that dedicates a chapter to this issue. Literature review effort revealed the benefits of qualitative pilot works and the role of this work in developing the main study.

PSs are much more common for quantitative studies than for qualitative ones (Crossman, 2007), and there are two possible reasons for this. First, a considerable amount of literature focuses on the benefit of PSs for eliminating any waste of financial funding by using inappropriate resources or instruments in the final study (Teijlingen \& Hundley, 2001; Crossman, 2007), and lab resources and instruments are rather more likely to be used in quantitative than in qualitative research. The second reason may lie in the flexibility of qualitative methodology. According to Creswell (2012), a flexible method, is required in studies exploring the human experience. This flexibility can help the researcher to tailor the research question(s) to the specific field of study. Additionally, data collection and analysis can be continually adjusted to the emerging findings. This flexibility typically exists in qualitative methodologies; if, for example, a researcher finds during an interview that the questions do not address their objectives, they can be refined spontaneously in the same interview. A qualitative final study can therefore often be pursued without a need for piloting it. 
On the other hand De Vaus (1993. p. 54) warns strongly: 'Do not take the risk. Pilot test first'. The existing literature advocates the importance of a PS from two perspectives: that of the research subject, and that of the researcher. From the perspective of the research, the implications of not piloting a study are highlighted by Teijlingen and Hundley (2002), who posit a possible case where problems existed with the research tool, and modifications had to be made in light of the findings from the PS. Without these modifications, the data would have been flawed or inaccurate. The question that needs to be addressed in a case where a study was not piloted, and aspects of the method needed to be modified within the research process (i.e. during the sampling of participants), is thus: "What is the base or road map for this modification?" The answer is that a PS can be this roadmap - according to Thabane et al. (2010) it can assist in defining the research question and to test the feasibility, reliability and validity of the proposed study design. Frankland and Bloor (1999, p. 154) similarly assert that piloting provides the qualitative researcher with a 'clear definition of the focus of the study', which helps the researcher to concentrate their data collection on a narrow spectrum of projected analytical topics. This benefit is further discussed below in the researcher's reflection after the PS (see 2.6 Reflection on the Pilot Study).

As to the perspective of the researcher, Teijlingen and Hundley (2001) explain that the nature of qualitative data collection tools such as interviews are often progressive, and that second or subsequent interviews in a study should be better than previous ones as the interviewer gains insights that are used to improve interview schedules and specific questions. For example, a qualitative interviewer conducting 15 focus group interviews will listen to the recordings or read through the transcripts of the first three or four in order to improve questions and ways to introduce issues to the group, or even add new topics for discussion. Although there is no specific PS in this case, analysis of earlier focus group interviews may thus help to improve later ones.

Some scholars have therefore argued that separate PSs are not necessary in qualitative approaches (Holloway, 1997). On the other hand, Janghorban et al. (2014) stress the importance of PSs for novice qualitative researchers to minimise the risk of encountering unmanageable problems while obtaining data, as well as in conducting the processes of data analysis and interpretation. Another benefit for novice researchers is that piloting qualitative approaches can also be carried out if the researcher 'lacks confidence or is a novice, particularly when using the interview technique' (Holloway, 1997, p. 121). Hence, even in qualitative research, where human experience is investigated and direct interaction between the research and the researcher is involved, PSs are considered highly useful for novice researchers. The question that needs to be addressed, however, is what benefit there might be in the case of an expert qualitative researcher exploring a novel intervention or an innovative application or method. I believe that in this context, too, a PS can help refine data collection plans with respect to both the content of the data and the procedures to be followed. The benefits of PSs for a researchers' experience and professional competence are consequently, important for both novice and expert researchers. 


\subsection{Challenges of Pilot Studies}

The following section discusses the two main questions that a researcher might confront when conducting a PS, namely (1) whether pilot participants are to be included in the main study or completely different participants should be recruited, and (2) whether data from the PS should be included in the main results, or instead disregarded. Teijlingen and Hundley (2001) discuss these two concerns for researchers in general, with the main focus on quantitative research. As this paper mainly focuses on qualitative PSs, the discussion in the following section is directed towards qualitative research.

\subsection{Conducting Interviews with the Same Participants in the Main Study}

Including the same participants in both pilot and main studies or including different participants in the main study is a significant question, especially if the selection criteria are narrow and finding suitable participants might thus be difficult or time-consuming. Teijlingen and Hundley (2001) answer this question for quantitative research, and do not favour the idea of conducting interviews with the same participants in the main study, since this might affect the sample size, as there will be difficulty to find number of participants twice (pilot and final study), especially if participants in the final study are supposed to come from the same clusters (e.g. schools, prisons or hospitals). Researchers can therefore conduct a sub-group analysis to assess to what extent the process of piloting influences the size of the intervention effect. On the other hand, using the same participants in both studies, also has advantages - according to Janghorban et al. (2014), conducting interviews with the same participants creates familiarity between the researcher and the participants, allowing both of them to behave more naturally later in the main research study. However, the issue with this claim, which involves asking the same person the same question twice, even though it might be in two different settings, is that it may lead to them losing interest in the study, in a phenomenon known as "semantic satiation". This psychological phenomenon involves the temporary influence that may be caused by the repetition of a word or phrase - according to Jakobovits and Lambert (1962), repetition may lead to loss of meaning and interest for the listener. To engage the participants in a qualitative study it might therefore be more beneficial to conduct the final study with different participants from those who have contributed to the PS; even though of course but both groups should share the same background (i.e. education, culture) depending on the study context and the research question.

Another point that needs to be considered with regards to the study sample is that PSs are nearly always based on a small number of participants, which means that the reliability of PS findings may be limited, even though they offer some indications of the likely size of the response rate in the main data collection (Teijlingen \& Hundley, 2001). According to Connelly (2008), extant literature suggests that a PS sample should be $10 \%$ of the sample size planned for the final study. This, however, is much more of an issue in a quantitative study, since the number of participants in the final study can be more than double of the number on the pilot study. This is usually different in qualitative studies, where the entire research sample can be a very small number of participants in homogeneous groups (Guest et al., 2006), and thus the impact of the final sample size is not expected to be very great. 


\section{Macrothink

\subsection{Including Data from the PS in the Main Study}

The second issue, that of including data from the PS in the main study, discussed in the literature mainly with regard to quantitative data. According to Peat et al. (2002, p. 57), 'an essential feature of a pilot study is that the data are not used to test a hypothesis or included with data from the actual study when the results are reported'. The obvious concern is that if there are problems with the research tool and modifications have to be made in light of the findings from the PS, data obtained in the PS could be inaccurate or faulty. However, where an established and validated tool is being used, and the PS is only meant to test other methodological aspects such as recruitment rates, it could be argued that such data would still be of value. In terms of data from qualitative studies, there are in most cases no drastic changes in the main study design after the PS, and since in this case data corruption is rather less of a concern, researchers often use some or all of their pilot data as part of the main study.

\section{Example of a Qualitative PS}

\subsection{Study Background}

The $\mathrm{PhD}$ thesis for which this PS was conducted is titled "Perceptions of online tutors about SCL in the Egyptian HE". It is a phenomenological qualitative study which uses both one-to-one interviews and focus groups as data collection tools. Data in the PS was collected from members of the Faculty of Education in one of the older universities in the north of Egypt. Data was collected in Arabic as the native language of Egyptians, and then translated into English by professional and accredited translators. The process by which the translation was done was back-translation to recheck the accuracy of the translation to improve its trustworthiness.

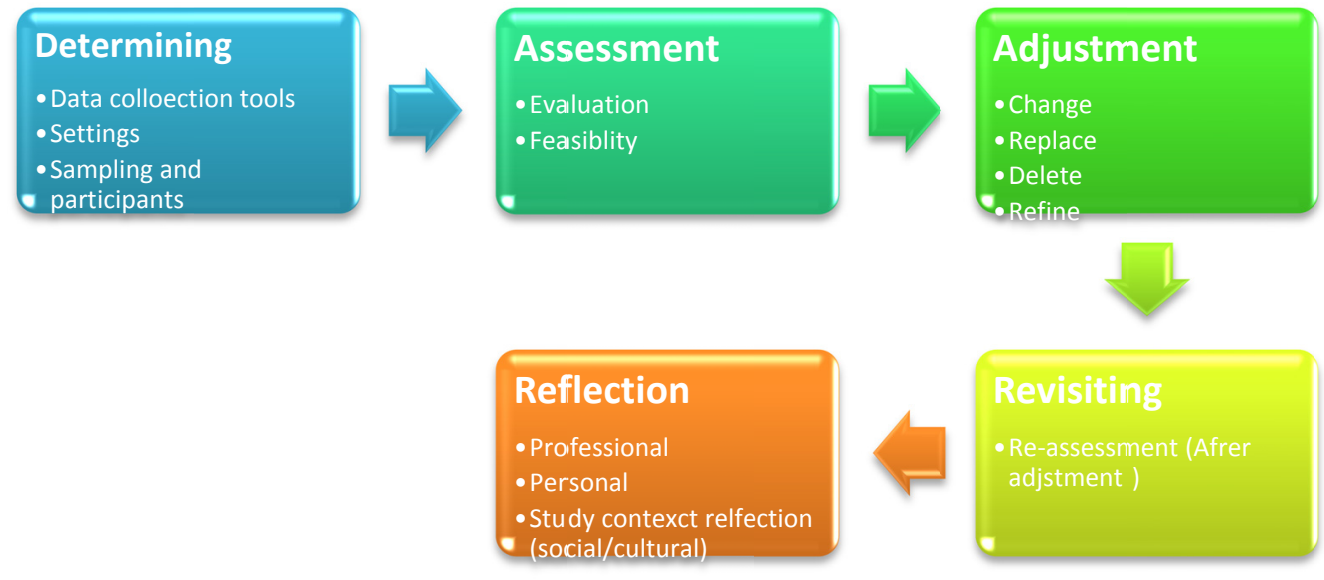

Figure 1. Steps of pilot study plan

The diagram (Figure 1) illustrates the suggested steps of a PS plan, starting with the determination of the PS plan including all aspects of the applied method and methodology. This is followed by an assessment of the tools based on their feasibility (i.e. by evaluating interview questions), and after this the adjustments of these tools and other settings of the 
research process were made. Next, the plan was revisited, meaning assessed again as to tools and techniques. At this stage, there might not be another opportunity to examine the study before the final data collection, and it is therefore suggested to prepare a list of questions in the assessment step to be answered and tested in the revisiting step. Finally, the lessons learned from the whole PS were reflected on. This reflection can be used in further research to avoid duplicating similar mistakes or to refine similar situations.

\subsection{Initial Plan for Piloting}

The PS for this particular PhD thesis research lasted two weeks. It was conducted through one-to-one semi-structured interviews and focus group interviews with 5 out of a total of 20 online tutors who had been selected as participants for the main study. The focus group interviews lasted for nearly an hour, and each one-to-one interview lasted between 45 and 60 minutes. The focus group was conducted on one day, and the individual interviews were held on the following few days, with only 2 interviews on the same day. All interviews and focus groups were audio- and video-recorded, the latter due to the plan to use a Facial Analysis Coding System (FACS). Interviews and focus groups were held after an informed consent form from the participants had been obtained, which covered issues such as confidentiality and the right to withdraw. It is worth mentioning that the time lag between the different interviews helped in obtaining feedback from the participants, who were asked to reflect on the entire discussion and then offer their opinions on the questions asked. Consequently, this feedback could be reflected on and used in the following interviews - an example of this reflection will be explained in section 2.6 Reflections on the Pilot Study.

My first visit to the faculty was a non-official meeting with participants. The purpose of this non-official meeting was to introduce myself to them and explain the purpose of my research. In this meeting, I emphasised our common ground and shared experiences to create a friendly atmosphere as suggested by Hurn and Tomalin (2013), who mention that especially in Arabic countries what is is 'getting to know the members of the other negotiating team, thus establishing trust and helping to break the ice' (p.178). A researcher from a different country can subject to numerous curious questions and enquiries - examples of the questions I was asked at the beginning of the focus group included "Why would a university in another country be interested in our perception towards a certain issue in education?" and "Is it acceptable to reveal the truth and declare a problem in our education system in front of others who may not have this problem at all? "Clarifying issues such as these with the participants can help to break the ice and enrich the data collected.

\subsection{Changes in the Initial Piloting Study Plan}

Because of the actual circumstances encountered at the location, a number of changes had to be made to the initial PS plan, including

1. The number of participants in the focus group was increased to 6, as a lady participant was hesitant to join the focus group for cultural reasons, then she decided to join.

2. The focus group and interview setting was moved to a small classroom which was away from the noise of the main buildings and was available at the requested times. 
3. Because of changes in the tutors' timetable, there was no individual day available for the non-official meeting only; instead it was conducted as an hour-long early-morning meeting on the same day as the focus group. During this meeting I was presented to the tutors, and we had tea together before starting the focus group.

Each of these changes had implications for the flow of the PS which will be discussed below.

\subsection{Initial Interview Questions}

The table below (Table 1) lists the initially planned interview questions, as well as the changes made to them after the PS. The reasons for the individual changes, additions and deletions of questions) are explained in the following section.

\section{Table 1. Initial interview questions}

1. As an online tutor, what are the differences between your teaching methods and strategies in the face-to-face environment and the Online Environment (OL)?

2. Describe your teaching style when working in an online setting.

Changes: Q2 is merged with Q5 (See details below the table)

3. What are your strengths for your position as online tutor?

Changes: Q3 is deleted and replaced by Q1 (see details below the table)

4. What are the challenges that online tutors may face in teaching?

Changes: Q4 is reworded and reordered (see details below the table)

5. What planning do you undertake before teaching an online class?

Changes: Q5 is merged with Q2 (see details below the table)

6. Tell me about the materials you use in online teaching.

\section{Changes: Q6 is Deleted (see details below the table)}

7. How would you handle a student who is not engaged in the course?

Changes: adding: give examples from your real teaching practices

8. How would you handle a student who is not self-motivated?

9. Rate the students' average ability to do the following :

Time management - self management- self-discovery- guided discovery- self evaluation

Note: rating is from $1-5$ ( 1 is the lowest and 5 is the highest)

10. How do you guide your students in their online search, within the studied topic? Give an example.

11. How do you encourage your students to be creative?

12. If one of the students suggested a new topic to be included in the course syllabus that was not directly related to the main topic, how would you respond to this?

13. What is your definition of empowering learners? Give examples where you seem to empower your learners while teaching them.

14. What are the benefits of empowering learners?

15. What are the concerns about empowering learners?

16. Which aspects of control do you give your learners? Give an example.

Changes: adding: give examples from your real teaching practices

17. What have you done to improve your knowledge as an online tutor in the last few months/ years?

18. How often do the training courses dedicated to online tutors you have attended take place? How would you evaluate them?

19. How ready do you think Egyptian Higher Education Institutes are for OL? 
Changes: Q19 adding another question (see details below the table)

20. Regarding Egypt, describe any constraints that can be obstacles to applying any of the topics we have discussed.

Changes: reordering the question (see details below the table)

21. Questions added about authoring the online content

\section{Question 2}

Question 2 was merged with Question 5 to be: "What plans do you make before teaching an online course? Give examples of your teaching styles such as using multimedia, games, simulations etc."

\section{Question 3}

Question 3 was deleted and replaced by question 1, which is more generic and less personal question. The reason behind this was a matter of cultural perspective - in general, in Middle Eastern culture it may not be completely acceptable to declare one's personal strengths with regards to careers and professionalism, since one could be accused of arrogance if one discusses one's points of strength. Conversely, talking about personal weaknesses may negatively affect a professional evaluation report or lead to missing an opportunity to pass a job interview. Writing on this issue in Arab culture, Al Suleimany (2009, p.402) also notes that "[i]f you are honest, sincere and genuine you just get ignored. Actually, you may be targeted and remarked for special raw and rough treatment." Consequently, the dominant cultural belief is that being completely honest and truthful is not an effective behaviour at your work place, but that one rather has to sometimes hide one's feelings or pretend to feel something else to get noticed and promoted. After the PS, answers to Question 3 ("What are your strengths for your position as online tutor?") were very short and unclear, and as a result in the final study Question 3 was replaced by Question 1, where the interviewees highlighted the affordances of online tutoring as compared to face-to-face tutoring in general.

\section{Question 4}

The question was reworded into one asking the interviewee to give an example of a challenge which they have confronted within teaching. Q 20, where the participants were asked about such challenges on a national level, was moved up in order to follow this question.

\section{Question 6}

Question 6 was deleted without replacement. Due to the limited technological resources in Egypt (Abdullah \& Albadri, 2010), most tools and programmes that were mentioned in the PS were basic and identical, and answers were lacking in rich information. It is noteworthy that although this PS was conducted in 2014, the limitation of resources noted by Abdullah and Albadri (2010) four years earlier still existed, though were gradually decreasing.

\section{Question 19}

This question was changed to be followed by a new question asking to give more details about missing resources or facilities that the online tutors have been confronted with, and which were 
considered to be obstacles for doing their jobs.

\section{Question 20}

This question was moved up in order to follow Question 4 since in answering Question 4 the participants tended to start by talking about personal challenges and then, unprompted, moved on to talk about the challenges on the national level.

\subsection{Summary of Changes in Questions after the Pilot Study}

Table 2. Summary of changes in questions after the PS

\begin{tabular}{ll}
\hline Question number & Change \\
\hline Question 2 & Merged with question 5 \\
Question 3 & Replaced by question 1 \\
Question 4 & Reordered \\
Question 6 & Deleted \\
Question 7 & Followed by another question \\
Question 16 & Followed by another question \\
Question 19 & Followed by another question \\
Question 20 & Reordered \\
\hline
\end{tabular}

With reference to the diagram in Figure 1 (see 2.1 above) the final interview questions were part of the adjustment stage where applied methods were adjusted (changed/deleted/added/replaced). The next step, the re-assessment, is quite similar to the assessment stage, the only difference being that the initial assessment is based on the fundamental study structure and original research plan while the re-assessment is based on the findings of the PS.

\subsection{Reflections on the Pilot Study}

This is the final stage of the PS plan (see Figure 1 in 2.1. above). The main challenge that I confronted in the PS was how to position myself as a novice researcher:. "The location and position of researchers influence the research process and therefore necessarily affect the final product", warn Thapar-Björkert and Henry (2004, p.365). In their view the researcher -researched relationship in fieldwork influences the research process and therefore necessarily affects the final research product. Heeding their warning about the researcher's location, I used the idea of the 'Six Thinking Hats' invented by De Bono (1999). These "hats" are used to look at decisions from a number of different perspectives, forcing us to move outside our habitual thinking style and helping us to get a more rounded view of a situation. While collecting my data, the "hats" I used were those of "researcher", "student", "colleague" (of the interviewees) and "social advisor". These "hats" inspired me to perform these different roles and helped me to think clearly and thoroughly by directing my thinking attention in one direction at a time. I found that in a qualitative study where human experience is investigated and direct interaction between the researcher and those researched is encountered, the PS helps researchers to position themselves and "wear the right hat" prior to final data collection. 


\section{Macrothink}

The following section discussed different challenges (i.e. ethical, cultural, social and professional) that were encountered in the PS, and how applying different research locations and "hats" helped me to respond to and reflect on each challenge. The challenges and my reflections on them discussed in the following section are:

1. Audio/video recording

2. Respect for the Tutor

3. Classroom setting

4. Questions to the researcher

5. Overriding of male gender in the focus groups

6. Questions with no answer

7. Learning from the interviewees' answers

8. Avoiding bias in research

\subsubsection{Audio/Video Recording}

Audio/video recording was an issue especially for female interviewees, who hesitated to share information in the focus group discussion and the interview after they had been informed that audio or video recording would be used. This hesitation turned into outright disapproval in two cases because of their conservative social and cultural perspective. In this situation, it was necessary for me to build a bridge of trust between myself and the participants, and to explain how highly privacy and confidentiality of data are valued in the university regulations. Here, the "researcher hat" was used to reassure the participants.

\subsubsection{Respect for the Tutor}

Due to cultural perspectives involving the issue of respect for a tutor there was a high wall between myself and the interviewees. This wall became obvious in the participants' habit of raising hands before commenting and even standing up to answer questions. This behaviour, it was explained to me, has its roots in an old saying in the culture, which I could clearly see is still applicable today: "Stand up for the tutor and respect him, the tutor is nearly a prophet." (Abdallah \& Albadri, 2010, p.17). My solution to this situation was to position myself as a colleague, meaning as just another online tutor towards the interviewees, and assure them many times that I was their colleague and would like to share common experiences in our mutual careers. Here the "colleague's hat" was used.

\subsubsection{Classroom Setting}

Another cultural challenge I was confronted with occurred due to the location of the interviews in a classroom setting. The consequence of this setting was that the interviewees did not interact and discuss freely, which became obvious in the lack of gestures regarding proxemics and kinesics. As a result, for the final data collection, I was keen to exchange the classroom for another place on the university campus such as a meeting room with a round table.

\subsubsection{Questions to the Researcher}

There were a number of questions asked by the interviewees, including "Why would a university in another country be interested in our perception towards a certain issue in 
education?", "Is it acceptable to reveal the truth?", "Is it acceptable to declare a problem in our education system in front of others who may not have this problem at all?", or "Can we say that.....?" followed by silence and hesitation to complete the statement.

Confronted by these questions I felt that we had exchanged roles, and that the participants were now the interviewers and I was an interviewee who had to give the best possible answer or risk failure. Here I used the "hats" of "researcher" and "social advisors". My answers to the participants focused on three main aspects:

- The reassurance that we all have weaknesses and we tend to try to work on eliminating them.

- It is important to exchange knowledge and experiences (by reminding them of events in the history of Egyptian education where it was in its best situation due to exchanging knowledge and sending delegates to other nations).

- The existence of globalisation processes that involve geographically disparate people whose ideas, knowledge and technologies are disseminated by a variety of technological means over vast distances.

\subsubsection{Overriding of Male Gender in the Focus Groups}

An issue that arose while moderating the pilot focus group was that the women felt they were not being fairly treated in matters of recruitment, selection, training and promotion (Al-Suleimany, 2009). The impact of this issue on the interviewees became obvious in a number of ways, including

- Head nodding and acceptance without discussion

- Disagreement without evidence

- Discussions becoming heated due to a difference in gender rather than in point of view

The "tutor's hat" and "social advisor's hat" were used in this situation. It is worth mentioning that in cases were controversial topics came up, the discussion was not easy to moderate.

\subsubsection{Questions with No Answer}

There were two questions in the interview with no clear answers or with only generic answers given by the participants. These questions were:

•"What are your strengths for your position as an online tutor?" and

•"What are your weak points for your position as an online tutor?"

Here I applied some of the reading I had done on the topic of transparency in Middle Eastern culture. (See 2.2 Initial Plan for Piloting, question 3)

In response to this I adapted my questions in the interviews and focus groups in a way that could bypass this issue. The questions were thus modified as follows:

- As an online tutor, what are the differences between your teaching methods and strategies in the face-to-face environment and the OL environment?"

•"Regarding Egypt, describe any constraints you may confront as an online tutor." 
In this situation, I used the "researcher's hat" to try to find reasons that would explain why these questions did not get answers, and to adapt the questions so that they would.

\subsubsection{Learning from the Interviewees' Answers}

As a researcher, I learned that it is possible to modify any question in the interview in response to the interviewees' answers. For example, I moved all the questions which focused on challenges and barriers in OL to the end of the interview or focus group meeting - it emerged during the PS that talking about the affordances at the beginning helped the interviewees to think creatively and effectively, while talking about the challenges at the beginning led them to assume that a situation was out of their control and that there was nothing they could do to change it.

\subsubsection{Avoiding Bias in Research}

Another skill I gained during the PS was how to minimise bias in research. One of the questions that I asked in the interview was "What is the best ratio between online learning and face to face learning?" One interviewee's answer was: "I do not see that online learning is effective at all. I believe that all learning resources have to be hard copies. OL is wasting our students' time and ruining their creativity skills." Since my own research is on the topic of OL, I naturally have a rather different point of view from that of the interviewee. If I have difficulty recognising that other people think differently than I do about a given topic or situation, this may lead me to comment on the interviewee's view or ask a biased question.

The interviews in the PS taught me firsthand that it is important to understand bias and how it affects research results and that I need to be aware of my own bias and how this may be expressed. In this situation, I used the "researcher's hat".

\section{Recommendations from the Pilot Study}

My reflections from the PS had different implications from both a professional and a personal perspective. The main lessons I have learned are summarised here.

- It is a good idea to follow questions the interview/focus group with a simple example which explains the question and clarifies the meaning and the angle that I wanted to approach for the participants.

- Before the PS I had considered two different approaches with regards to translating the Arabic data into English. The first was to start by translating the entire scripts first, and then conducting the analysis of the data from the translated scripts. The second was to conduct data collection, transcription and analysis all in Arabic, with only the final findings translated into English. By the end of the PS, I had recognised that my main references and academic literature were in English, and concluded that it would be easier to start by having the entire scripts translated from Arabic to English, and conduct the rest of the research (data analysis, findings and discussion) in English.

- In the PS focus group I had to be skilful with probing questions in situations where the discussion moved away from the main topic. In those cases, I had to choose a word or a statement from the discussion and rephrase it into a question. Though these questions 
may not be listed in the original questions script, they helped to answer the research questions without losing the evolving discussion.

- When watching the recording of the first interview, I observed that I had been less than cautious about revealing some of my preconceived ideas. In the following interviews I was therefore rather more cautious not to reveal any of my prior knowledge of and views on the topic.

- I analysed the non-verbal data from the interviews using the audio/voice recording. The analysis for the PS was for one participant only to experiment with the quality of analysis and evaluate the time and effort necessary. Analysing one participant's interview in the PS using FACS resulted in the knowledge that the analysis of just that one participant's interview with FACS was 8 hours. Because of time limitation, it was agreed by me and my supervisors to use Interpretative Phenomenological Analysis (IPA) only rather than including FACS for the purposes of the $\mathrm{PhD}$.

- The location of the interviews is important - interviewing in a classroom did not help to break the ice with the participants and it did not help me to wear any "hat" except of the researcher and tutor, so that I learned that it would be more beneficial to change the interview setting from the classroom to a more social setting, such as a social space on the campus. This change in venue also helped with the analysis of the non-verbal communication in the research, as setting in comfortable chairs with a low table leads to the exchange of more information than the kind of formal setting. (Verma, 2005)

- In terms of the theoretical framework, the PS confirmed that the use of constructivism and social constructivism were the appropriate theories that could support my study, as they define the kinds of variables that I wanted to look at.

- In my research proposal and the methodology chapter the plan was to follow a thematic data analysis. However, after collecting the data of the PS, it was found that using IPA would be a more appropriate method to analyse the data collected in this study

- After the PS it was decided to reduce the number of participants from 30 to 20 , since the collected data turned out to be rich, and the time allocated or data collection and analysis would not have allowed me to collect data from more than 20 participants.

- The participants highly appreciated receiving a gift at the end of the interviews - after collecting the data that I gave the participants a pen with pictures of famous historical places from the UK as a souvenir. Although these gifts were not expensive at all (approximately 50p each), the present had a positive effect on the participants and was much appreciated.

\section{Summary of the Pilot Study}

In conducting the PS a number of issues from professional, social, ethical, and cultural perspectives, were identified. The perspectives themselves had two different facets, the first being the researcher's point of view which involved asking questions and encountering participants. The second was from the point of view of the participants' and how they interacted with the study design and research questions. The PS helped me to reduce the risk of errors or problems and to identify and resolve as many potential problems or issues as possible. More specifically, the PS helped me to draw a clear road map for the research including data collection tools, methodology, data analysis and theoretical framework. The PS also helped me 
to understand that it is necessary to read up on the culture of the participants even though I myself am part of this culture. Furthermore, the PS helped me to learn how to achieve the study goals and objectives through the use of a variety of "thinking hats" during the data collection stage. From a professional point of view as a researcher, checking the participants' awareness of instruments used for collecting data (such as focus group discussions) is important, as participants in the study might not be familiar with some of these research tools. Finally, as a novice qualitative researcher, conducting a PS helped to train me into becoming a flexible researcher - I learned to keep in mind that the questions I am asking about my topic would change slightly, which I had not been expecting. I learned about being reflexive, placing myself and my research practice under examination to aid me in acknowledging any ethical dilemmas (such as the male gender overriding the female gender) that might permeate the research process and could hinder the acquisition of knowledge.

Acknowledging only limited benefits of PSs for research only narrows down their uses. PSs can help with the research process itself, leading to remarkable implications which last until the very end of the final study, and the researchers themselves add to their research experience and career competence. It is, however, worth considering the expected benefits from a PS before conducting one, as there might be cases where only a few benefits might be achieved, while in other studies many benefits may be achieved from the PS. What needs to be kept in mind in either case, though, is the advice of Thabane et al. (2010), who stress that PSs should be welldesigned with clear feasibility objectives, clear analytic plans, and explicit criteria for determining success of feasibility.

\section{Conclusion}

A PS is crucial for a well-planned study design. Its benefits are unarguably and essential for both qualitative and quantitative researchers, if, that is, the PS has a well-defined set of aims and objectives to ensure methodological rigour and scientific validity. This paper has focused on piloting qualitative data collection methods used by a $\mathrm{PhD}$ researcher in the field of Education. According to the results discussed in this paper, the researcher had different experiences and confronted different challenges while conducting the PS with a view to numerous perspectives, including ethical, cultural, social and professional. The PS informed the feasibility and identified necessary modifications in the design of the final $\mathrm{PhD}$ research. This paper thus concludes that there are multiple benefits to conducting a PS, and that there is a need for more discussion among researchers on both the processes and outcomes of PSs. The experience shared in this paper offers clearer guidance to researchers who are new to using PSs in qualitative research. However, there are still a number of unresolved issues and conflicting opinions on PSs that need to be addressed, such as the questions on whether to use the same participants, setting and data in both pilot and final study. These issues can be a focus of further research. The main message of this paper for conducting research is therefore: "Learn from your (and other researchers') mistakes."

\section{References}

Abdallah, S., \& Albadri, F. (2010). ICT Acceptance, Investment and Organization: Cultural Practices and Values in the Arab World. New York: Hershey. 
Al-Suleimany, M. S. N. (2009). Psychology of Arab Management Thinking. Canada: Victoria. $\mathrm{BC}$.

Connelly, L. M. (2008). Pilot Studies. Medsurg Nursing, 17(6), 411-2. PMID: 19248407

Crossman, A. (2007). Pilot Study, An Overview, Social sciences. Retrieved July 11, 2017, from https://www.thoughtco.com/pilot-study-3026449

Baker, T. L. (1994). Doing Social Research (2nd ed.). New York: McGraw-Hill.

Creswell, J. W. (2012). Qualitative Inquiry and Research Design: Choosing Among Five Designs (3rd ed.). Thousand Oaks, CA: Sage.

De Bono, E. (1999). Six Thinking Hats, New York: Back Bay Books.

De Vaus DA. (1993). Surveys in Social Research (3rd ed.). UCL Press: London.

Frankland, J., \& Bloor, M. (1999). Some issues arising in the systematic analysis of focus group material. In R. Barbour, \& J. Kitzinger (Eds), Developing Focus Group Research: Politics, Theory \& Practice. Sage: London.

Guest, G., Bunce, A., \& Johnson, L. (2006). How many interviews are enough? An experiment with data saturation and variability. Field Methods, 18(1). https://doi.org/ $10.1177 / 1525822$ X05279903

Holloway, I. (1997). Basic Concepts for Qualitative Research. Oxford: Blackwell Science.

Hurn, B. J., \& Tomalin, B. (2013). Cross-Cultural Communication: Theory and Practice. UK: Palgrave Macmillan.

Jakobovits, L. A., \& Lambert, W. E. (1962). Semantic satiation in an addition task." Canadian Journal of Psychology, 16, 112-19. https://doi.org/10.1037/h0083238

Janghorban, R., Latifnejad, R., \& Ali Taghipour, A. (2014). Pilot Study in Qualitative Research: The Roles and Values. Journal of Hayat, 19(4), (ISSN 3-2014).

Kim, y. (2010). The Pilot Study in Qualitative Inquiry Identifying Issues and Learning Lessons for Culturally Competent Research. Qualitative Social Work, 10(2), 190-206. https://doi.org/10.1177/1473325010362001

Padgett, Deborah K. (2008). Qualitative Methods in Social Work Research (2nd ed.). Los Angeles, CA: SAGE.

Peat, J., Mellis, C., Williams, K., \& Xuan, W. (2002). Health Science Research: A Handbook of Quantitative Methods, London: Sage.

Prescott, P. A., \& Soeken, K. L. (1989). The potential uses of pilot work. Nursing Research, $38,60-2$.

Sampson, Helen. (2004). Navigating the Waves: The Usefulness of a Pilot in Qualitative Research. Qualitative Research, 4(3), 383-402. https://doi.org/10.1177/1468794104047236 


\section{Macrothink}

Teijlingen, V., \& Hundley, V. (2001). The Importance of Pilot Studies. Social Research. Retrieved August 15, 2017, from http://sru.soc.surrey.ac.uk/SRU35.html

Teijlingen, V, E., Rennie, A. M., Hundley, V., \& Graham, W. (2001). The importance of conducting and reporting Pilot Studies: the example of the Scottish Births Survey. Journal of Advanced Nursing, 34, 289-295. http://doi.org/10.1046/j.1365-2648.2001.01757.x

Teijlingen, V., \& Hundley, V. (2002). The importance of pilot studies. Nursing Standard, 16(40), 33-6. http://dx.doi.org/10.7748/ns2002.06.16.40.33.c3214

Teijlingen, V. E., Rennie, A. M., Hundley, V., \& Graham, W. (2002). The Role of Pilot Studies in Midwifery Research. Midwives: The Official Journal of the Royal College of Midwives, 5(11), 372-374.

Thabane, L., Ma, J., Chu, R., Cheng, J., Ismaila, A., Rios, L., Robson, R., Thabane, M., Giangregorio, L., \& Goldsmith, C. (2010). A tutorial on Pilot Studies: the what, why and how, BMC Medical Research. BMC Medical Research Methodology. https://doi.org/10.1186/1471-2288-10-1

Thapar-Björkert, S., \& Henry, M. (2004). Reassessing the Research Relationship: Location, Position and Power in Fieldwork Accounts. Social Research Methodology, 7(5), 363. http://dx.doi.org/10.1080/1364557092000045294

Verma, S. (2005). Body Language Your Success Mantra: Your Success Mantra. New Delhi: Educa Books

\section{Copyright Disclaimer}

Copyright for this article is retained by the author(s), with first publication rights granted to the journal.

This is an open-access article distributed under the terms and conditions of the Creative Commons Attribution license (http://creativecommons.org/licenses/by/3.0/). 